Supporting Information

\title{
Van der Waals bound Organic/2D-Insulator Hybrid-Structures: Epitaxial Growth of Acene Films on hBN(001) and the Influence of Surface Defects
}

\author{
Darius Günder ${ }^{a}$, Kenji Watanabe ${ }^{b}$, Takashi Taniguchi ${ }^{c}$, Gregor Witte ${ }^{a, *}$ \\ a Fachbereich Physik, Philipps-Universität Marburg, 35032 Marburg, Germany \\ ${ }^{\mathrm{b}}$ Research Center for Functional Materials, National Institute for Materials Science, Tsukuba 305-0044, Japan \\ c International Center for Materials Nanoarchitectonics, National Institute for Materials Science, Tsukuba 305-0044, Japan \\ *E-Mail: gregor.witte@physik.uni-marburg.de
}

\section{Contents}

1. Identification of $h \mathrm{BN}$ azimuth directions and crystal habit

2. Morphology of ultrathin acene films on hBN crystals

3. Emission spectrum of the halogen lamp

4. Absorption spectra of acene multilayer films with different molecular orientation

5. Additional optical micrographs of exfoliated $h \mathrm{BN}$ flakes and $h \mathrm{BN}$ crystals covered with acene films

6. Schematic representation of hBN step edges and step faces 


\section{Identification of $h \mathrm{BN}$ azimuth directions and crystal habit}

a)

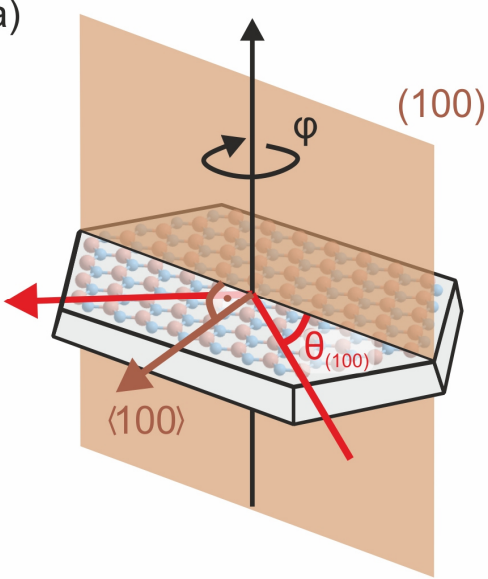

b)

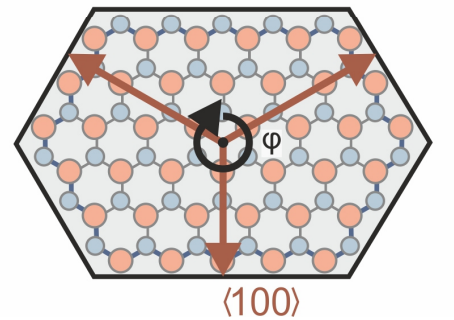

c)

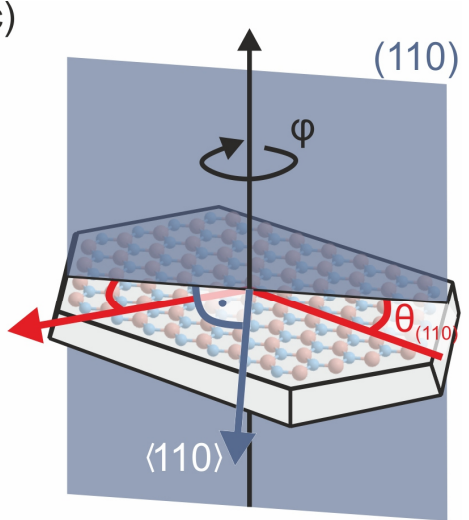

d)

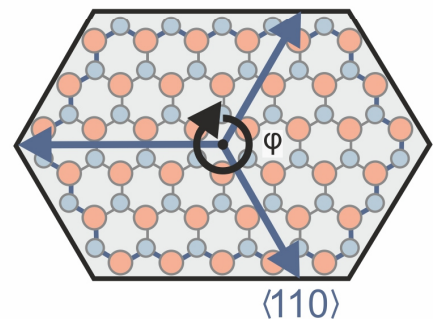

Figure S1: Schematic visualization of the measurement geometries used for the azimuthal in-plane XRD scans of (a) the $(100)_{h \mathrm{BN}}$ plane and (c) the $(110)_{h \mathrm{BN}}$ plane. The directions perpendicular to the probed planes define the $\langle 100\rangle_{h \mathrm{BN}}$ and $\langle 110\rangle_{h \mathrm{BN}}$ azimuths shown in (b,d)

To identify the azimuth directions of the $h \mathrm{BN}(001)$ surface and correlate them with the habit of the single crystals in-plane XRD measurements were performed. For this purpose the sample was aligned such that the first order diffraction of the $(100)_{h B N}$ plane $\left(\theta_{100}=20.8^{\circ}\right)$ is obtained at grazing incidence, and subsequently the azimuthal distribution of this reflex is measured in a $\varphi$-scan, yielding the $\langle 100\rangle_{h \mathrm{BN}}$ azimuth (which is perpendicular to the $(100)_{h \mathrm{BN}}$ plane). The analogue procedure was also performed for the $(110)_{h \mathrm{BN}}$ planes. 

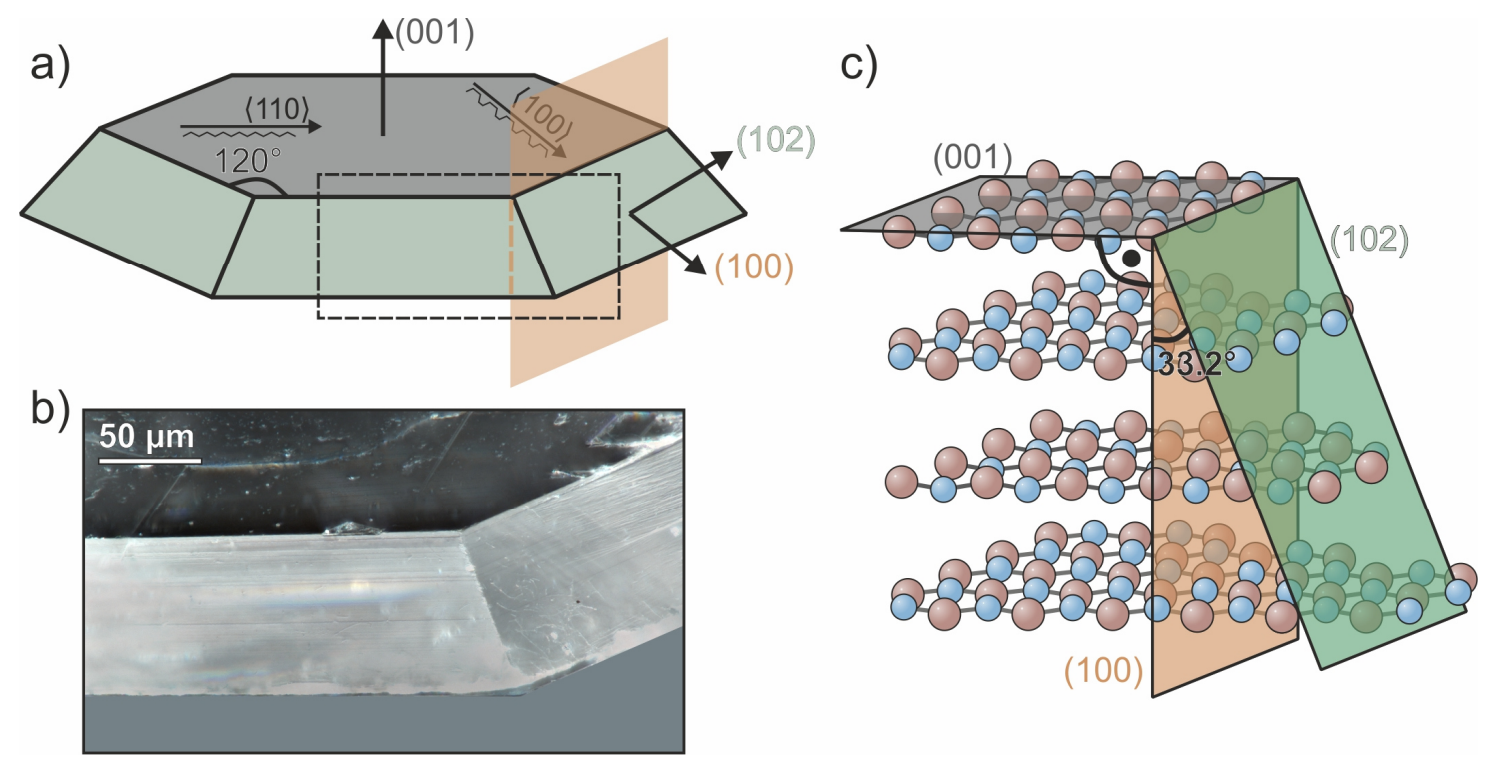

Figure S2: (a) Schematic representation of the $h B N$ single crystal habit, which is compared with (b) an optical micrograph showing a corner that is indicated by the dashed region in (a). (c) Schematic model of the (102) side face.

In Figure S2 (a) the habit of a $h \mathrm{BN}$ single crystal is depicted. The crystal sides are not spanned by $\{100\}_{\mathrm{hBN}}$ planes, which are perpendicular to the (001) $)_{\mathrm{hBN}}$ surface plane as it can be observed in Figure S2 (b) where a magnified optical micrograph of a $h B N$ crystal corner is shown. The image was obtained by recording several individual micrographs of varying focused sections, which allow to construct an overall focused micrograph and to determine height and angular information. This analysis shows that the crystal side faces are tilted about $(35 \pm 5)^{\circ}$ with respect to the $(100)_{\mathrm{hBN}}$ plane and can be well approximated by the $(102)_{\mathrm{hBN}}$ plane, which is tilted by $33.2^{\circ}$ relative to the $(100)_{\mathrm{hBN}}$ plane as depicted in the structural model in panel (c). The crossing line between the basal surface plane and the side faces corresponds to the high symmetry $\langle 110\rangle_{\mathrm{hBN}}$ (zig zag) direction. 


\section{Morphology of ultrathin acene films on $h B N$ crystals}

a)
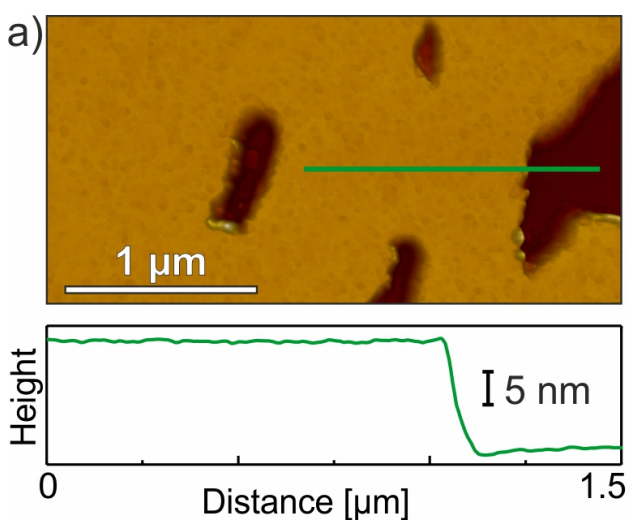
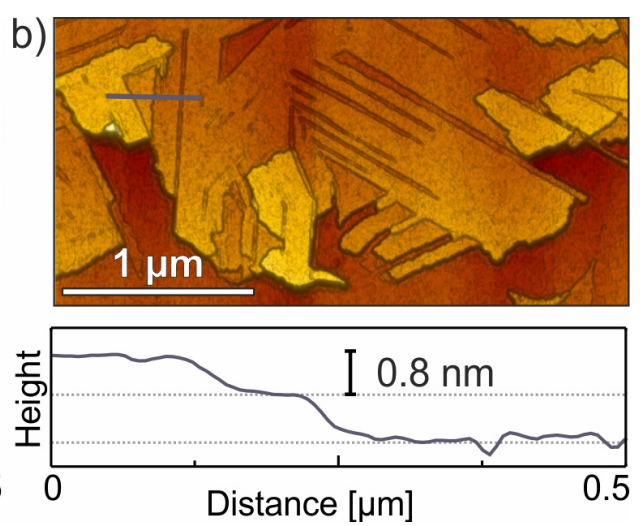

Figure S3: AFM micrographs showing the morphology for the initial stage of film growth for 3nm (a) PFP and (b) PEN films on hBN single crystals, together with topographic line scans.

In addition to thick acene film (cf. Fig. 2b,c) also the morphology of 3nm thick films was studied by means of AFM to characterize the initial stage of film growth on $h \mathrm{BN}$ single crystals. In both cases molecularly flat regions are formed. Although PFP forms much larger islands than PEN, the PFP films reveal characteristic holes as well as only partial wetting of the $h \mathrm{BN}$ crystal surfaces resulting in compact islands that coexist with uncovered surface regions. The morphology of thin PEN films is very similar to that of thicker films and exhibits elongated islands with molecularly flat terraces. Since the overall roughness of the thin films is distinctly smaller than for thick films (cf. 100nm films shown in Fig. 2c), molecular steps can be resolved. They reveal a characteristic height of about $0.8 \mathrm{~nm}$ which can be associated with the double layer spacing of the (022) oriented PEN films of the Siegrist phase. 


\section{Emission spectrum of the halogen lamp}

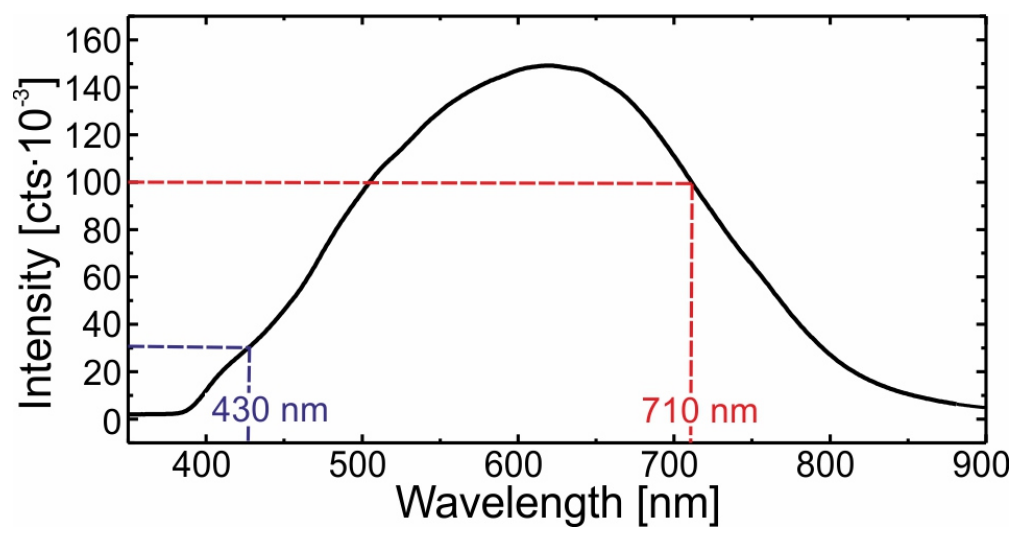

Figure S4: Emission spectrum of the halogen lamp used for the polarization resolved optical microscopy and absorption measurements. The marked regions denote the energy of the characteristic L-polarized (blue, 430nm) and M-polarized (red, 710nm) absorption bands of PFP.

Although PFP molecules exhibit two distinct absorption bands with different polarization in the probed spectral region (L-polarized at $430 \mathrm{~nm}$ and M-polarized at $710 \mathrm{~nm}$ ), a distinct polarization contrast is observed for the reflectivity. This can be understood, if one considers the different spectral brightness of the used lighting lamp which is about three times more intense at $710 \mathrm{~nm}$ than at $430 \mathrm{~nm}$. This explains that the observed polarization contrast is dominated by the M-axis polarized absorption band. 


\section{Absorption spectra of acene multilayer films with different molecular orientation}
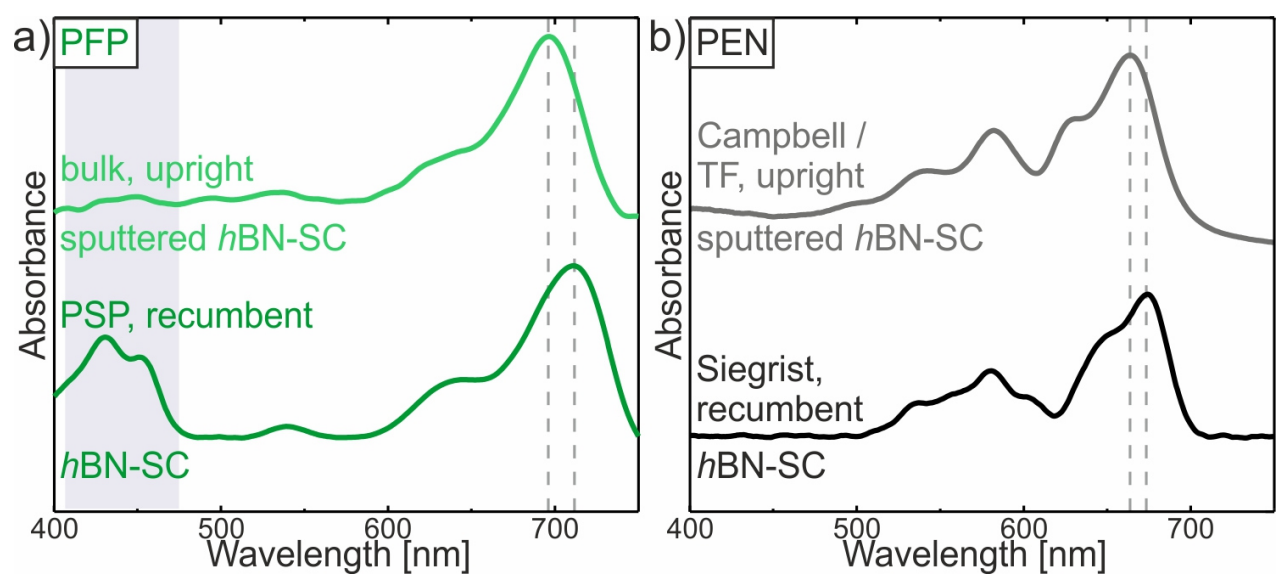

Figure S5: Unpolarized UV/Vis absorption spectra of (a) PFP and (b) PEN multilayer films with different molecular orientation and different crystalline phases grown on $h \mathrm{BN}$ crystals.

Figure S5 compares the characteristic absorption spectra of crystalline PFP and PEN multilayer films grown on $h \mathrm{BN}$ single crystals of different surface quality. As discussed in the main paper on intentionally sputtered $h B N$ surfaces acenes grow with an upright molecular orientation. Here, PFP films adopt the bulk phase, while PEN films adopt the Campbell phase in coexistence with the thin-film (TF) phase. By contrast, on highly ordered exfoliated $h \mathrm{BN}$ crystals both acenes reveal a recumbent molecular orientation and PFP crystallizes in the $\pi$-stacked polymorph (PSP), while PEN adopts the Siegrist phase polymorph. The different molecular orientations manifest in the presence or absence of the L-polarized absorption band of PFP. In addition, the different polymorphic phases also exhibit small but notable differences in the Mpolarized absorption bands, since the excitonic excitation energy depends sensitively on the molecular packing within the crystalline films. In case of PFP this yields low energetic excitations at 712nm for the PSP-phase and at $697 \mathrm{~nm}$ for the bulk phase [S1]. A similar effect is also found for PEN [S2] and yields excitonic excitations at $674 \mathrm{~nm}$ for the Siegrist phase and at $665 \mathrm{~nm}$ for the TF/Campbell phase.

[S1] Rinn, A.; Breuer, B.; Wiegand, J.; Beck, M.; Hübner, J.; Döring, R.C.; Oestreich, M.; Heimbrodt, W.; Witte, G.; Chatterjee, S. Interfacial Molecular Packing Determines Exciton Dynamics in Molecular Heterostructures: The Case of Pentacene-Perfluoropentacene ACS appl. Mater. Interfaces 2017, 9, 42020-42028.

[S2] Meyenburg, I.; Breuer, T.; Karthäuser, A.; Chatterjee, S.; Witte, G.; Heimbrodt, W. Temperatureresolved optical spectroscopy of pentacene polymorphs: variation of herringbone angles in single-crystals and interface-controlled thin films Phys. Chem. Chem. Phys. 2016, 18, 3825-3831. 


\section{Additional optical micrographs of exfoliated $h B N$ flakes and $h B N$ crystals covered with acene films}
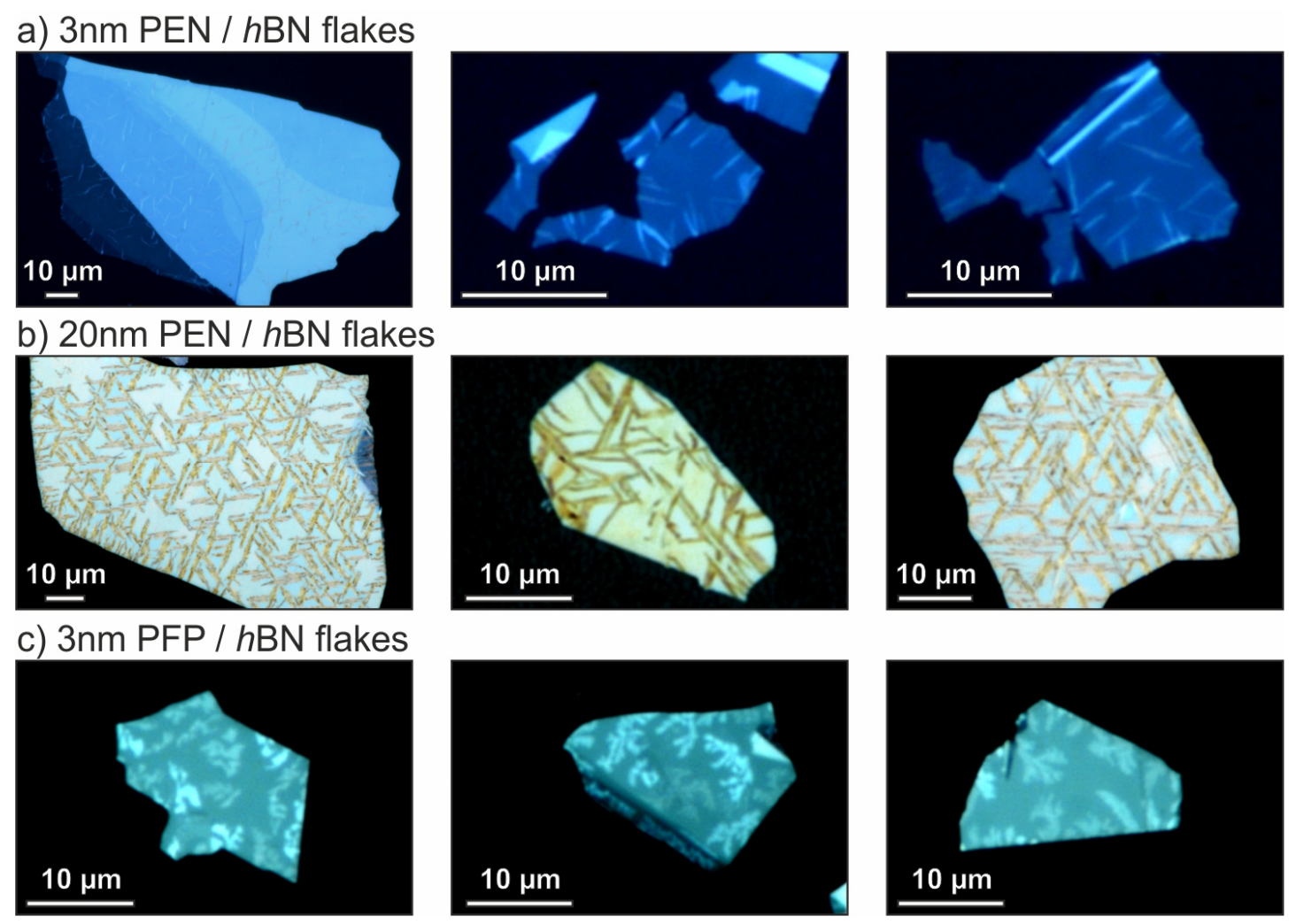

Figure S6: Additional optical micrographs of exfoliated $h \mathrm{BN}$ flakes covered by acene films: (a) 3nm PEN, (b) 20nm PEN, (c) 3nm PFP.

Due to its simplicity, exfoliation from van-der-Waals bonded bulk materials is a widely used approach to prepare 2D flakes. However, the surface quality of these flakes can vary significantly. In order to be able to make a characteristic statement about film growth on exfoliated flakes, we have examined more than 30 flakes in this study. Figure $\mathbf{S 6}$ shows representative optical micrographs of exfoliated flakes on which thin acene films have been grown. Evidently the observed morphology is very similar as characteristic feature such as the PEN fibers are found on all samples. Note, that all considered flakes were further analyzed by AFM and micro-spot absorption measurements and only representative results are discussed in the main paper. 

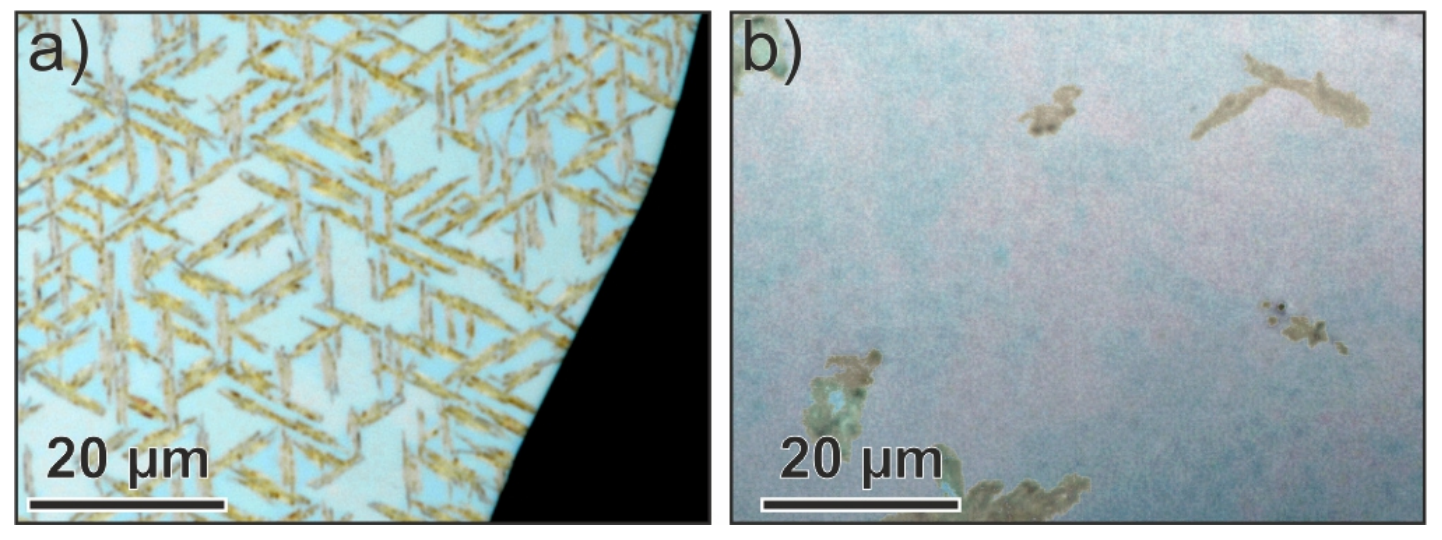

Figure S7: Optical micrographs of a nominally 20nm thick PEN film grown on (a) an exfoliated hBN flake and (b) on a non-appropriately exfoliated hBN crystal.

Optical micrographs of a nominally 20nm thick PEN film grown on hBN substrates of different step edge density are depicted in Figure S7. As already discussed in the main paper hBN step edges of flakes caused by the exfoliation process lead to a formation of elongated PEN fibers as it is represented in Figure S7(a). In some rare cases the same type of fibers can also be observed on hBN crystals as depicted in Figure S7 (b) which is caused by a non-appropriate exfoliation of the hBN crystal before film preparation. Note, that the overall fiber density is much lower compared to the PEN film on an exfoliated hBN flake indicating that less hBN step edges are induced on hBN crystals than on exfoliated hBN flakes. 


\section{Schematic representation of hBN step edges and step faces}

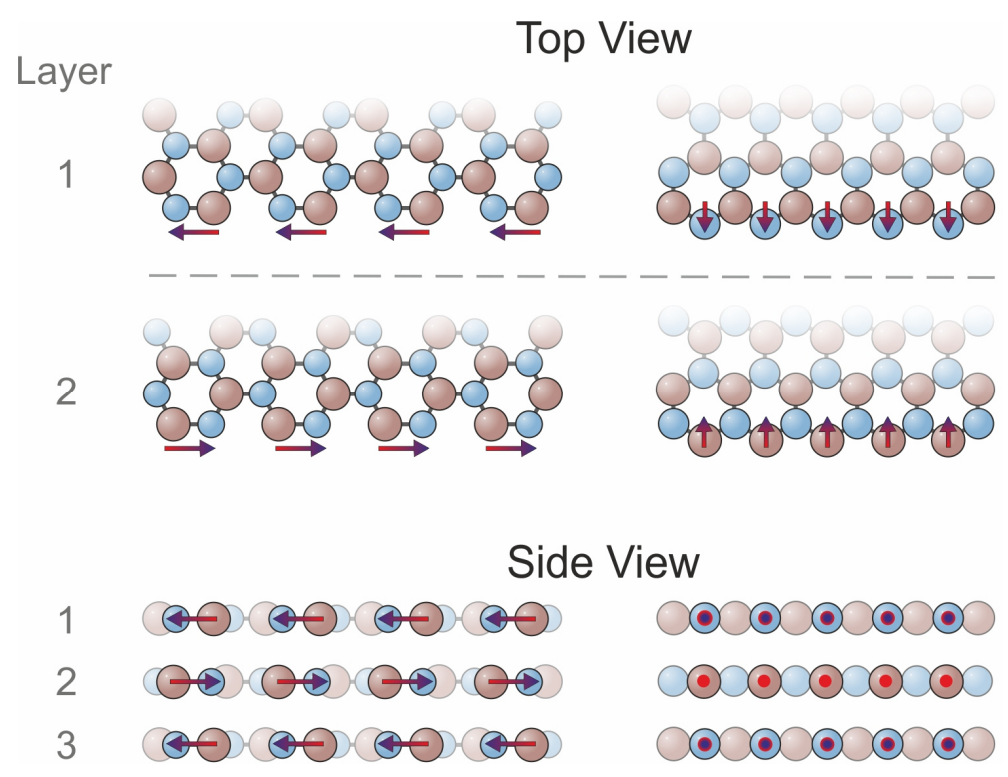

Figure S8: Comparison of the arrangement of polar BN-bonds (indicated by arrows) at the different step edges (and related step faces) on $h \mathrm{BN}$, shown for $\langle 100\rangle_{\mathrm{hBN}}$ oriented steps (i.e. armchair) on the left-hand side and for $\langle 110\rangle_{\mathrm{hBN}}$ steps (i.e. zigzag) on the right-hand side, as a top view onto the basal plane and side views onto side faces of an ideal bulk truncation.

As a consequence of the different electronegativity of boron and nitrogen the BN-bonds are polar, which leads to distinct difference in the polarity and charge of the steps. Figure $\mathbf{S 8}$ compares the situation for the different hBN step edges. While armchair steps (lhs) are terminated by equal amount of boron and nitrogen atoms they are electrically neutral, the zigzag steps (rhs) are charged. The depicted cut along the (100) plane yields an alternating arrangement of B- and N-terminated edges. However, it should be noted that this step edge (and likely also the step face) is less stable and might undergo a reconstruction. 